Ann. Génét. Sél. anim., I970, 2 (2), I35-I38

\title{
PROPORTION MENDÉLIENNE ANORMALE POUR LE GÈNE « COU N CHEZ LA POULE DOMESTIQUE
}

\author{
P. MÉRAT \\ avec la collaboration technique de L. Durand \\ Station centrale de Génétique animale, \\ Centre national de Recherches zootechniques, 78-Jouy-en-Josas \\ Institut national de la Recherche agronomique
}

\section{RÉSUMÉ}

Un rapport de ségrégation anormal a été trouvé pour le gène $\mathrm{Na}$ ("cou nu ) dans une population de volailles. Dans le croisement $\delta^{\circ}{ }^{\circ} \mathrm{Na} n a \times$ 우 na na, on ne trouve que 43 p. 100 de "cous nus " parmi les poussins femelles. Le rapport de ségrégation est normal chez les mâles issus de ce croisement et dans les deux sexes pour le croisement réciproque. Une "pénétrance "incomplète du gène $\mathrm{Na}$ est très peu probable, et une mortalité plus élevée des filles $\mathrm{Na}$ na n'expliquerait ni la proportion des sexes qui s'écarte de l'égalité en sens contraire dans les deux classes phénotypiques, ni la proportion normale trouvée lorsque la mère est hétérozygote. Un phénomène de "fertilisation sélective " est donc vraisemblable.

\section{INTRODUCTION}

Le gène $N a$ (" cou nu ") est connu depuis longtemps chez la poule domestique. Son effet suppresseur du plumage en dehors des "ptérylies " est dominant; sa pénétrance complète, jointe à une identification facile dès l'éclosion, en fait un bon gène " marqueur " (HUTr, I949). L'apparition de proportions s'écartant, dans un croisement, des valeurs théoriques prévisibles nous a donc paru intéressante à signaler et à interpréter.

\section{MATÉRIEL ET MÉTHODES}

Les données sont extraites d'une population élevée à Jouy-en-Josas, où divers gènes à effet visible sont maintenus en ségrégation, pour une recherche d'associations entre ces gènes et des caractères quantitatifs. Le croisement entre mâle hétérozygote et femelle homozygote normale 


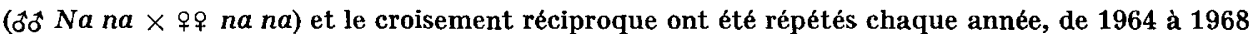
inclus. L'identification du phénotype "cou nu " ou "normal " dans la descendance était faite à l'éclosion et vérifiée à l'âge de 8 semaines; celle du sexe avait lieu à 8 semaines avec vérification deux semaines plus tard.

\section{RÉSULTATS}

Les proportions mendéliennes sur le total des années pour chaque croisement et par sexe figurent au tableau I.

TABLEAU I

Proportions mendéliennes relatives au gène "cou nu ", par sexe

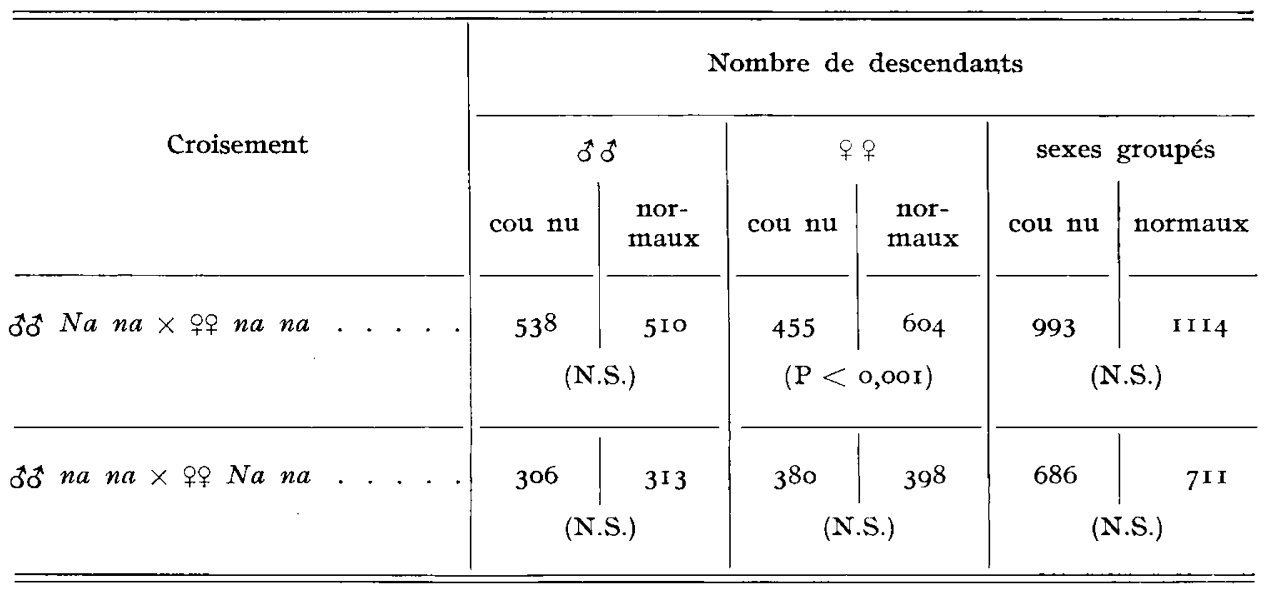

Les nombres observés ne s'écartent nulle part significativement de la proportion théorique $I / I$ dans le second croisement (mère hétérozygote), non plus que chez les descendants mâles lorsque le père est hétérozygote. Par contre, on constate alors un défaut important du phénotype "cou nu " parmi les filles : 43 p. roo au lieu des $50 \mathrm{p}$. Ioo attendus. Le $\chi^{2}$ correspondant est égal à 20,96 pour I degré de liberté $(\mathrm{P}<0,00 \mathrm{I})$ alors que sa valeur est de 0,75 chez les mâles. Le $\chi^{2}$ d'hétérogénéité entre sexes pour la proportion en cause est de 14,76 pour I d.1. $(P<0,00 I)$. L'anomalie constatée est donc bien limitée au sexe femelle.

L'explication par une pénétrance du gène " cou nu " incomplète seulement chez les femelles serait a priori peu vraisemblable. Elle paraît pouvoir être exclue par le résultat normal obtenu dans le croisement réciproque, les mêmes années et avec des géniteurs de même origine. De plus, parmi les filles de phénotype " normal " issues d'un père hétérozygote, 38 ont reproduit 1'année suivante avec un nombre de descendants en principe suffisant pour vérifier leur génotype : 9 d'entre elles, accouplées à des coqs " normaux " $n a$ na et ayant eu au moins Io descendants, n'en ont donné aucun de phénotype " cou nu "; 29 autres ont été accouplées à des coqs hétérozygotes $N a n a$ et ont eu au moins $1_{5}$ descendants. 
Pour toutes, le génotype le plus vraisemblable est na na. Le rapport de ségrégation dans leur descendance se conforme bien à cette hypothèse; par contre, ce rapport conduit, pour 22 d'entre elles, à un $\chi^{2}$ supérieur à 5 dans 1'hypothèse où elles seraient $N a$ na, la valeur de ce même $\chi^{2}$ étant comprise entre 3 et 5 pour les 7 autres.

$\mathrm{Au}$ total, aucune de ces 38 poules d'apparence " normale " ne parait être hétérozygote. Si l'on suppose donc que toutes sont bien na na, les chances d'obtention de ce résultat dans 1'hypothèse d'une pénétrance incomplète peuvent être évaluées en estimant à o,I4 la probabilité pour une poule $N a$ na d'être de phénotype "normal ". Cette estimation repose sur le fait que $43 \mathrm{p}$. Ioo environ de " cous nus" sont observés parmi les filles au lieu de $50 \mathrm{p}$. Ioo, la différence représentant $\mathrm{I} 4 \mathrm{p}$. Ioo de ces filles. A partir de cette valeur, la loi binômiale indique que la probabilité d'obtention de $3^{8}$ filles na na serait alors inférieure à $5 \mathrm{p}$. Ioo.

Une autre explication à envisager serait une mortalité embryonnaire ou postembryonnaire plus grande du génotype $N a$ na chez les femelles issues du croisement $\delta^{\star} \mathrm{Na} n a \times$ 우 $n a$ na. Plusieurs faits vont à l'encontre d'une telle interprétation. Tout d'abord, la proportion dans le croisement réciproque s'expliquerait difficilement ainsi; on vérifie non seulement qu'elle est normale, mais aussi qu'elle diffère significativement de celle observée dans le premier croisement (le $\chi^{2}$ d'hétérogénéité entre croisements pour la proportion chez les femelles est égal à 6,20 d'où $\mathrm{P} \simeq 0,0 \mathrm{I})$.

En outre, la considération séparée de la proportion des sexes dans chaque catégorie phénotypique permet de penser qu'il y a non seulement un défaut de filles " à cou nu " lorsque le père est hétérozygote, mais encore plus un excès de filles " normales " par comparaison avec le nombre de fils de la même catégorie. En effet, la proportion des femelles par rapport aux mâles est inférieure à l'unité chez les "cou nu" (455 contre 538, d'où $\chi^{2}=6,94, \mathrm{P}<0,0$ ) mais elle est aussi très nettement supérieure à I dans le phénotype normal (604 contre 5Io, d'où $\left.\chi^{2}=7,93, \mathrm{P}<\mathrm{o}, \mathrm{Or}\right)\left({ }^{1}\right)$. Seul le défaut de filles " cou nu " serait explicable par une viabilité réduite, mais non l'excès de filles à plumage normal.

Le taux d'éclosion et de survie des jeunes apparaît d'ailleurs normal et du même ordre, chaque année, que la moyenne du troupeau, tant dans le croisement ơ $\mathrm{Na} n a \times$ 우 na na que dans le croisement réciproque.

\section{DISCUSSION}

Nous sommes donc conduits à prendre en considération l'hypothèse d'une anomalie des proportions à la fécondation, puisque les phénotypes observés paraissent bien correspondre aux génotypes supposés, et qu'une sélection naturelle exercée sur les zygotes ne rend pas compte des faits d'une manière satisfaisante.

Du fait qu'elle est restreinte à un sexe dans un croisement où le père seul est hétérozygote, cette anomalie suppose une interaction entre le génotype du spermatozoïde $(\mathrm{Na}$ ou $\mathrm{na}$ ) et la constitution de l'ovule relativement à l'hétéro-

(') alors qu'au total elle est voisine de $\mathrm{r}$. 
chromosome ( $Z$ ou $W$ ). En d'autres termes, il s'agirait d'un phénomène de fertilisation sélective, les spermatozoïdes porteurs de $N a$ ou de na ayant une probabilité différente de féconder un ovule $W$.

Il est intéressant de souligner que nous avons déjà rencontré à plusieurs reprises, chez la poule, un phénomène de nature apparemment analogue, relativement aux loci $R$ (MÉRAT, I962, I963), $C$ (MÉRAT, I966 a) et peut-être $W$ (MÉRAT, I 966 b). Nous avons été conduit à suggérer que le stade où ce phénomène entre en jeu pourrait se situer au niveau d'une "compétition " entre noyaux spermatiques ayant pénétré dans le cytoplasme de l'ovule (MÉRAT, I969), la fécondation polyspermique étant normale chez les oiseaux (PIKo, rg6r).

Dans un autre article, nous mettrons en parallèle les différents exemples cités ci-dessus pour tenter d'en tirer une conclusion d'ensemble.

Rę̧u pour publication en février 1970.

\section{SUMMARY}

ABNORMAL MENDELIAN PROPORTION OF THE " NAKED NECK" GENE IN POULTRY

An abnormal segregation ratio was found for the gene $\mathrm{Na}$ (" naked neck") in a poultry strain. In the cross ${ }^{\alpha} \delta \mathrm{Na}$ na $\times$ 우 na na, only 43 per cent " naked necks" were found among female chicks. The segregation ratio was normal for males in the same cross and for both sexes in the reciprocal cross. An incomplete penetrance of the $N a$ gene is very unlikely, and a higher mortality of $\mathrm{Na} n a$ female progeny can explain neither the sex-ratio in the two phenotypic classes, which is deviated in opposite directions, nor the normal proportion found when the dam is heterozygous. The likely conclusion is therefore the operation of a " selective fertilization" phenomenon.

\section{RÉFÉRENCES BIBLIOGRAPHIQUES}

Hutr F.B., r949. Genetics of the fowl. Mc Graw Hill, N.Y.

MERAT P., r962. Ségrégation anormale pour les allèles " crête simple " et "crête en rose " chez la Poule. I. croisement ơ Rr $\times$ irr. Ann. Biol. anim. Bioch. Biophys., 2, rog-ri7.

Merat P., r963. Ségrégations anormales pour les allèles "crête simple " et "crête en rose "chez la Poule. IV. Discussion d'ensemble. Ann. Biol. anim. Bioch. Biophys., 3, I33-I 4 I.

Merat P., ig66 a. Segrégation anormale au locus C chez la poule domestique. Ann. Biol. anim. Bioch. Biophys., 6, 423-425.

MERAT P., I966 b. Irrégularités des proportions mendéliennes au locus $W$ chez la poule domestique. Ann. Biol. anim. Bioch. Biophys., 6, 249-254.

MÉrat P., 1969. Données complémentaires sur des anomalies de ségrégation au locus $\mathrm{R}$ chez la Poule, lorsque le père est hétérozygote. Ann. Génét. Sél. anim., 1, 227-235.

Prko I., I96r. Ia polyspermie chez les animaux. Ann. Biol. anim. Bioch. Biophys., 1, 324-384. 